\title{
IL BENE CULTURALE: L'IDENTIFICAZIONE E LA TUTELA, PROBLEMI QUALITATIVI E QUANTITATIVI
}

\author{
AMEDEO BELLINI (*)
}

SuNTO. - Alle sue origini la tutela si fondava sulla individuazione degli oggetti testimoni degli eventi fondamentali della storia, esemplari per caratterizzare il passato; la tutela ed il restauro erano lo strumento per la loro conservazione ma anche per recuperare il senso originario sopprimendo manomissioni, completando le lacune. L'ampliamento degli interessi conoscitivi, dai grandi eventi ai dati della vita comune e di cultura materiale, la coscienza della relatività degli esiti dell'indagine storiografica e del carattere soggettivo del giudizio estetico, del fatto che ogni evento od oggetto è plurisignificante e che quindi la sua rilevanza dipende dal sistema di relazioni nel quale lo si colloca, hanno condotto a considerare degni di interesse tutti i documenti di civiltà e infine tutto ciò che ha potenzialità di costituire esperienza di cultura. In questo senso "bene culturale" non è un insieme individuabile nel numero, né in particolari categorie di oggetti; è valore che dipende dalle "cose" ma soprattutto dal rapporto che si istituisce in circostanze variabili fra esse e le persone. I parametri di valutazione che conducono alla scelta di conservazione o di distruzione derivano dal riconoscimento di possibilità o impossibilità, da valutazioni che riguardano le esigenze vitali, quindi si collocano sul piano etico. La tutela si deve esercitare quindi preventivamente laddove si prendono decisioni di natura politica che ne determinano le condizioni di esercizio, solo successivamente come intervento. Occorre infine dare un ruolo fondamentale nell'economia alla produzione ed allo scambio di beni immateriali, tra essi in primo piano i servizi culturali.

$* * *$

ABSTRACT. - At its origin the tutelage was based on the detection of objects that were witness to fundamental events in history, exemplary for characterizing the past; restauration was the instrument for their conservation, but it was also used to recuperate their original sense, cancelling any tampering, and closing the gaps. The expansion of cognitive interests, from great events to common life data and material culture, the con-

(*) Istituto Lombardo Accademia di Scienze e Lettere. Politecnico di Milano, Italia. E-mail: amedeo.bellini@polimi.it 
sciousness of the relativity of the results of historiographical investigation and the subjunctive character of aesthetic judgement, the fact that every event or object has multiple meanings and therefore its relevance depends on the relation system in which it found, has lead to the consideration that all of civilization's documents are worthy of interest and finally all of that has the potential of creating a cultural experience. In this sense, "cultural heritage" is not a collection of items quantifiable in numbers, nor in particular categories of objects; it is a value that depends on the "items", but especially on the rapport that is established, under variable circumstances, between the items and people. The parameters of evaluation that lead to a choice of either conservation or destruction derive from the recognition of possibility or impossibility, the assessments concerning vital demands, and therefore these parameters are found on an ethical level. Tutelage must be exercised preventively where political decisions are made that determine the operating conditions, and only subsequently as intervention. Finally, a fundamental role must be given to the production and exchange of intangible goods in the economy, among these, in the forefront, cultural services.

I temi che ci pone il convegno, identificazione e tutela dei beni culturali, sono complessi e non certo esauribili in una giornata di riflessione che tuttavia può essere utile nel mettere a confronto considerazioni che si fondano su ben diversi punti di vista. Per affrontare il tema di una definizione di "bene culturale" può essere utile un cenno a quanto ci ha preceduto, in particolare fissando la nostra attenzione sul momento nel quale si è formata una tutela istituzionale, un'attività dello Stato destinata alla conservazione, al restauro di una parte di quanto ci è pervenuto dal passato come una affascinante, ma talora ingombrante, eredità morale. Ciò avviene a muovere dai primi decenni dell' 800 quando si forma l'idea romantica della storia come un processo progressivo, caratterizzante ed individualizzante, in opposizione all'antistoricismo illuminista. L'idea di una continuità tra passato e presente è spesso concepita come rapporto di causa ed effetto: appare quindi necessario, per comprendere l'oggi, studiare la storia e di conseguenza conservarne i documenti. In linea generale, è ciò che distingue dal precedente interesse verso le opere, non più restaurate per interessi antiquari o per un desiderio di completezza formale, ma come documenti da studiare e da comprendere nel loro messaggio. Analogamente a quanto avvenuto per i documenti scritti ci si interroga sul carattere veritativo di quelli materiali che vengono depurati dalle interpolazioni quando ritenute travisanti o incongrue, completandoli ove lacunosi, trasferendo su di essi i metodi, per quanto possibile, elaborati per l'analisi del documento scritto. E' esattamente quanto veniva definito "restauro". E' ancor più significativo rilevare come in Italia, 
comunque più di quanto sia avvenuto in altri paesi europei, esso abbia avuto significato, soprattutto quello dell'architettura ma non solo, come una attività legata alla scoperta delle fonti della storia patria, al riconoscimento dell'identità nazionale da parte di tutti; non a caso nell'Impero Austroungarico, realtà sovranazionale, erano state emanate dallo Stato disposizioni per la conservazione dei monumenti/documenti esplicitamente antirestaurative.

Questo ci ricorda che tutela e restauro, che ne è conseguenza diretta, non sono mai state operazioni politicamente neutre anche se il loro implicito significato in quella direzione non sempre appare noto agli operatori, soprattutto quando l'ideologia politica è mediata da quella delle concezioni estetiche.

L'acquisizione della relatività del valore documentario all'ambiente fisico e morale in cui l'opera si trova, del suo essere strumento di identità comunitarie locali, è una acquisizione lenta e abbastanza tarda, non tanto sul piano della comprensione del fenomeno culturale, quanto come traduzione da parte dello stato in pratica della tutela organizzata, ancora oggi in forma molto limitata. L'estensione nasce comunque in relazione a valutazione critiche che superano antiche preclusioni di ordine temporale (il barocco, il rococò) o riferite ad aree geografiche ma che comunque ritengono possibile individuare gerarchie di valore e di ottenere dalla ricerca storica una conoscenza sufficientemente sicura del passato da consentire di individuare i capisaldi testimoniali da tutelare.

Le posizioni attuali sono molto diverse da quelle ottocentesche la cui influenza, nell'ambito culturale di cui stiamo trattando, si è protratta a lungo: le novità di prospettiva introdotte dalla storiografia francese del primo novecento con lo studio della vita dell'uomo comune, dei dati di cultura materiale; il superamento della ottimistica fiducia nella possibilità di individuare una ratio univoca nel percorso della storia; le nuove basi del rapporto tra storia e storiografia fino alle tesi estreme che vogliono essere la prima il prodotto della seconda; l'idea che la ricerca storica metta in luce attendibili relazioni che hanno significato in relazione allo scopo dell'indagine ma da leggersi anche nelle condizioni di relatività determinate dalle intenzioni, dai limiti e dalle qualità del produttore, dalla qualità e quantità dei documenti sopravvissuti, in assenza di indicazioni anche minime sulle possibili modificazioni di prospettiva che potrebbero essere prodotte da ciò che manca; la questione, estremamente discussa anche recentemente, del rapporto tra il fatto e la sua interpretazione, fino alla coscienza lontana nel tempo, ma 
priva di conseguenze nella tutela, del carattere plurisignificante del documento e dell'oggetto e che quindi ciascuno di essi può avere valore fondamentale in una narrazione storiografica ed essere trascurabili in un'altra. Ciò tende ad escludere gerarchie di valore fondate su di un parametro privilegiato. Per esemplificare, ancora negli anni sessanta ed oltre si lamentava il carattere ideologico dello scavo archeologico e della sua restituzione in sito o museale che rappresentavano una città ed una società mostrandone i dati di eccezionalità, con una ricostruzione che era frutto di una concezione della storia che sembrava superata; ma dobbiamo anche registrare le ricostruzioni, i rifacimenti falsificanti che, nel settore del restauro pittorico, hanno tuttora una significativa presenza (forse un revival), mai abbandonati in architettura.

Una visione, quella moderna, dove accanto al senso delle gerarchie qualitative, valide all'interno dello stesso sistema di ordinamento, si affianca la coscienza della rilevanza dei dati quantitativi, del carattere indefinibile a priori del valore testimoniale: come la scomposizione delle fonti ha consentito di trarre conoscenze in settori ben diversi da quelli per i quali un documento era stato prodotto, come l'apertura di nuovi settori di interesse, o nuove scoperte, hanno determinato un riesame delle fonti scritte, altrettanto può avvenire per i documenti materiali. In sostanza ogni distruzione determina una riduzione delle possibilità di conoscenza non sostituibile con l'analisi dell'oggetto soppresso (come avviene nella raccolta dei dati caratterizzanti quanto rimosso nello scavo) o l'eventuale loro trasferimento su un altro supporto, certamente parziale, soprattutto inevitabilmente deficitario rispetto alle istanze conoscitive che nasceranno in futuro. Se ogni distruzione è perdita, è da evitare non soltanto quando essa sia motivata dal raggiungimento di obbiettivi venali, di fini di consenso politico, ma anche se discende da valutazioni apparentemente nobili come potrebbero essere l'affermazione di una grande costruzione intellettuale, di una poetica, o, avviene spesso, ai fini di una comprensione semplificata che soddisfi esigenze di massa, come accade, per esempio, nelle forme di restauro motivate dall'attirare turismo culturale (frequenti per iniziativa privata, ma non assenti dall'intervento pubblico, in genere chiamate "valorizzazione"). Sotto il profilo delle tutela delle testimonianze storiche l'unico atteggiamento teoricamente giustificabile è quello che si astiene da qualsiasi cancellazione, che garantisce la molteplicità delle letture, le potenzialità di quelle non immediatamente leggibili. Ovviamente ci sono cose non conservabili, per incapacità tecnica, per ragioni legate ad 
un modello di produzione e consumo che produce enormi scarti, per esigenze che derivano dalle qualità che si richiedono alle condizioni di vita, per la naturale azione del tempo. Ciò che può giustificare una eliminazione, sono dunque situazioni di impossibilità tecnica o esigenze vitali, e quindi motivazioni di natura etica. Nessun problema è risolto con questa impostazione, ma essa pone la questione sul suo terreno più corretto, anche se, ovviamente, considerare "bene culturale" tutto ciò che può determinare un'esperienza intellettuale o sentimentale, il riconoscimento di sé in un oggetto esterno che produca correlazioni coinvolgenti, costituisca momento della memoria o richiamare altro alla memoria, pone difficili problemi di individuazione del limite tra legittime scelte personali ed atti non accettabili per ragioni di interesse collettivo. Dobbiamo però essere consapevoli che oggi la "difesa" di molte opere di "grande rilevanza" storica (o artistica) è resa vana da fenomeni di natura economica, politica, molto più che da scelte consapevoli, dalla padronanza di strumenti critici per quanto complessi e condivisi essi siano nell'azione di tutela. Comunque occorre sempre ricordare che quella dei beni culturali è una categoria ben più ampia di quella delle cose d'arte così come comunemente vengono intese. Ma bisogna anche osservare che leggi di tutela, applicabili ad un numero definito di cose, e non potrebbe essere diversamente, sono per questo stesso fatto insufficienti a salvaguardare tutto ciò che ha interesse, e quindi devono integrarsi in un complesso più ampio di norme, di atteggiamenti, di fini perseguiti con strumenti i più diversi.

Gli ostacoli alla tutela provengono soprattutto da azioni economiche, naturalmente con maggiore rilevanza nel caso dell'architettura, del paesaggio (non più naturale ma sempre antropizzato) ma non mancano i danni anche alle opere destinate alla fruizione contemplativa diffuse sul territorio, cioè nel più ampio "museo" contestualizzato possibile, che si presentano comunque come oggetti complessi nei materiali, nelle lavorazioni, modificati nel tempo, spesso attraverso restauri, densi di valori documentari non figurativi, e solo di questi ultimi spesso la tutela si occupa in via esclusiva. L'opera "d'arte" è comunque un oggetto che ha una propria storia, che appartiene alle esperienze di vita, che è soggetto ad una molteplicità di giudizi appartenendo a molte correlazioni di pensiero, a una molteplicità di eventi, a meno che non si voglia sostenere che si tratti di una realtà spirituale ontologicamente distinta dagli oggetti di comune esperienza, ma in questo ambito di pensiero si tratterebbe di una "creazione" che assai raramente si realizza, ed il tema 
della sua tutela dovrebbe essere trattato (come si è giustamente teorizzato a muovere da quella concezione) come un caso a sé del tutto particolare.

Non è opportuno qui ricercare cosa sia l'opera d'arte, un dibattito infinito, mi limito a richiamare alcune definizioni diverse da quella indicata qui sopra: c'è chi l'ha definita, in una delle fasi del suo pensiero teoretico, come l'espressione più elevata del lavoro umano, e questo consentirebbe di individuare una gerarchia; altri l'hanno semplicemente e fenomenologicamente definita come ciò che ha avuto un particolare riconoscimento della coscienza e si tratta dell'assunto che ha il maggiore riscontro nei documenti ufficiali della tutela, ma anche la più disattesa. Nel linguaggio corrente, ma anche in sedi culturalmente qualificate, è frequente l'attribuzione di valore d'arte a ciò che, si comprende dal contesto, è giudicato realizzazione di particolare qualità espressiva ma anche oggetto eseguito con particolari abilità tecniche, spesso contaminando i significati. Oggi si è attribuita la qualità "arte" anche ad avvenimenti, manifestazioni che possono dissolversi senza lasciare tracce, o lasciarne ma del tutto prive di un nesso fisico con ciò che le ha prodotte. Si ricordi il grande compositore Carl Heinz Stockhausen che definì l'attentato alle torri gemelle di New York la più grande opera d'arte mai prodotta, ma anche la presentazione come tale di un viaggio in cui l'artista aveva lasciato particolari segni del suo percorso, subito smentito da altro che lo tacciava di essere superato, vecchio, rivendicando la propria opera: un percorso che non aveva lasciato alcuna traccia.

Abbandonando ad altri l'onere della definizione voglio far osservare che dal punto di vista della tutela ritenere l'opera d'arte come ciò che ha ottenuto una particolare considerazione dalla coscienza, definizione che sta alla base anche dell'ultimo documento ufficiale sulle modalità di restauro e che trova consonanze con riflessioni estetiche recenti, è suscettibile di dilatare in modo potenzialmente illimitato la categoria degli oggetti di interesse "artistico". Innanzitutto il riconoscimento dell'opera assegna un ruolo al fruitore (anche se la considerazione del carattere attivo della sua presenza nella costituzione della realtà dell'opera, del suo accrescimento, come produttore di un pensiero che, se noto, modifica i modi della percezione, risale almeno agli inizi del secolo scorso), sopprime il senso dell'opera d'arte come valore assoluto ed immutabile, accetta e legittima il dato che ciò che è apparso tale in un'epoca può non esserlo più in un'altra, e ciò riguarda interi periodi storici; accetta il fatto che opere rifiutate in un certo momento siano 
rivalutate in un altro, ma, e questo è fondamentale, con una lettura che muta i parametri di riferimento; la relatività del giudizio estetico va ben oltre il giudizio soggettivo individuale. Il discorso si potrebbe estendere alla condizione moderna che vede il rapido apparire di tendenze e il loro "superamento" immediato, con una rapidità che aveva già sconcertato Hans Tietze e con lui tutti i critici della gloriosa scuola di Vienna. L'assunzione di un parametro ristretto di tutela, la disattenzione verso ciò che in un certo momento appare un disvalore, non è quindi accettabile. Bisogna ancora rilevare come certa arte contemporanea, quella astratta, quella concettuale, per esempio, abbiano abbandonato qualsiasi rapporto con la rappresentazione della natura, ciò significa che la comprensione avviene soltanto attraverso un percorso intellettuale: senza la conoscenza di ciò che ha preceduto l'opera, del senso che essa ha all'interno di polemiche, discussioni, prese di posizione, affermazioni teoriche; è raro che la pura visione, la percezione del colore, di una struttura spaziale, risultino emozionanti e comunque si avverte che quell'aspetto non è esaustivo. Tuttavia non si tratta di una condizione nuova: la conoscenza di un'opera può limitarsi all'apprezzamento del messaggio immediato, come potrebbe avvenire per una persona proveniente da una civiltà lontana o primitiva di fronte ad un capolavoro, o superare questa condizione grazie ad un insieme di conoscenze, cioè ad una formazione culturale; ma anche chi, pur appartenendo alla nostra civiltà, ignora in tutto o in parte il messaggio iconografico o iconologico, l'ambito concettuale in cui l'opera è prodotta, ciò che ha preceduto l'artista, il suo percorso mentale, oppure, in altro ambito, la struttura architettonica, non ha una comprensione piena; ben diversa è l'emozione di chi apprezza concetti, scelte tecniche, evoluzione dei modi espressivi, rapporti con il contesto sociale al momento della realizzazione, nel tempo successivo, nella stratificazione di pensiero critico che si è espresso ed è stato acquisito dai successivi fruitori. L'apprezzamento, il giudizio che ne derivano, sono risultato di un processo complesso in cui l'esperienza esistenziale ha una funzione fondamentale (l'esperienza "estetica" è una particolare "esperienza storica"?). Ancora ricordiamo che, viceversa, la trasposizione di un opera in un contesto diverso da quello della sua produzione può dare valore d'arte ad oggetti d'uso comune o prodotti per motivi assai diversi da quelli che indichiamo a base della produzione artistica: il caso più noto è quello dell'apprezzamento delle statue lignee e delle maschere provenienti dall'Africa presso molti movimenti di avanguardia del primo Novecento, il Cubismo in 
particolare. Una serie di osservazioni che ci deve rendere cauti anche nel giudizio verso quelle produzioni dozzinali che affollano i mercatini settimanali: a parte la loro rilevanza da un punto di vista sociologico a partire dal quesito di ciò che sia comunemente considerato arte, di ciò che soddisfa il sentimento estetico nelle sue fasi più elementari, dobbiamo riconoscere che ciò che muove molte persone all'apprezzamento di quanto i "colti" disprezzano è lo stesso sentimento che muove ciascuno di noi: riconoscere qualcosa della nostra persona, della nostra esperienza in un oggetto esterno. È l'atteggiamento proprio anche del più raffinato critico che, pur aderendo a consolidate gerarchie di valore, ha infine una serie di valutazioni che corrispondono alla sua personalità, ai suoi interessi culturali più coinvolgenti, anche in campi diversi da quelli della sua prevalente attività, che lo portano ad avere un singolare piacere nella contemplazione di opere "minori". Quale possa essere la rilevanza nei processi di riconoscimento di sé degli oggetti più comuni, di ciò che anche minimalmente ha fatto parte della nostra esperienza di vita, ci è stato spiegato con grande maestria da Remo Bodei.

Un paio di esempi riassuntivi: in occasione del $150^{\circ}$ anniversario dell'unità d'Italia è stata pubblicata dall'Istituto dell'Enciclopedia Italiana una storia della letteratura che non si basa su di un giudizio di qualità letteraria ma sull'influenza esercitata nella formazione della coscienza nazionale: scompaiono i grandi scrittori ed appaiono nomi ritenuti in genere secondari, popolarmente molto apprezzati; potremmo fare la stessa cosa per pittura, scultura ed architettura ed otterremo risultati altrettanto sorprendenti. La prospettiva storica è mutata; l'emozione che ci produce la lettura orientata da questo nuovo punto di vista è molto diversa da quella dell'apprezzamento della capacità espressiva? Non è forse vero che la musica di Giuseppe Verdi trova presso la generalità degli "intenditori" e dei critici contestazioni che tendono a negargli (ovviamente con eccezioni) un "valore musicale assoluto" ma il nostro piacere nell' ascolto non dipende anche da quanto sappiamo della rilevanza che la sua figura ha avuto nella formazione della coscienza nazionale nella vicenda risorgimentale? Nella capacità della sua musica di essere coinvolgente? Nella sua aderenza totale al senso del melodramma, rappresentazione popolare per eccellenza che pure affascina l'intelletualità?

Il problema della identificazione dei valori non è quello di costruire gerarchie consolidate per individuare criteri selettivi, ma piuttosto quello di riconoscere i valori propri di ciascuna testimonianza, 
semplice o complessa che sia; è chiaro che ogni intervento di tutela o di "restauro" dovrebbe tendere alla permanenza di tutte le possibili letture del documento, realtà sempre complessa, mai derivare da una ideologia, da una lettura "critica", da un'ipotesi storiografica, da un sentimento estetico personale.

Poiché è ovvio che non è proponibile una conservazione totale di ciò che ci ha preceduto (sarebbe un'impresa paragonabile a quella sulla quale Umberto Eco scrisse un memorabile divertimento letterario: costruire una mappa del mondo in scala uno a uno) è opportuno ricordare che quanto qui si propone è una tesi limite che richiede però si dia luogo ad un mutamento dei parametri di valutazione. Non tuteleremo ciò che non siamo capaci di conservare per incapacità tecnica, per obiettivi problemi che deriverebbero dalla accumulazione indiscriminata, per esigenze di qualità della vita, che possono obbligare a manomissioni importanti soprattutto nell'architettura e negli assetti delicatissimi del territorio, per scelte fondamentali di natura economica. Spostare il campo delle decisioni dalle valutazioni ideologiche a quello dello "stato di necessità", degli interessi vitali, dalle propensioni formali travestite da estetica a valutazioni in definitiva di natura etica, non risolve problemi, non elimina dubbi, non annulla scelte soggettive soprattutto dove il confine tra bisogno oggettivo, bisogno psicologico, legittima affermazione dell'io, hanno confini labili, ma pone la discussione su di un piano più corretto, quello etico.

Da questo punto di vista è bene sottolineare che troppo spesso si pone in campo una falsa contrapposizione tra conservazione e valorizzazione che non ha ragione di essere: la prima ha come fine la diffusione della conoscenza, in termini non superficiali, che comporta quindi quelle indagini analitiche che consentono di indentificare le peculiarità dell'oggetto. Non sarà mai lecita una modificazione che divenga funzionale alla diffusione di una falsa coscienza del bene, che sia soddisfazione di una domanda di massa, espressa da persone a cui non sono stati forniti mezzi per una comprensione di adeguata complessità. Ricerca per la comprensione dell'identità; attività per conservare i dati materiali che trasmettono conoscenza, che preservano le peculiarità, incrementare le capacità di capire, anche attraverso l'uso consapevole, questo ci sembra possa essere un corretto procedimento di "valorizzazione".

Se poi osserviamo quanto di fatto avviene dobbiamo riconoscere che l'attività di tutela è "vittima", per così dire, dei processi economici che corrispondono spesso ad interessi privati, non sempre legittimi, che 
vengono solo talora contrastati, con esiti non sempre brillanti. Di questa insufficienza possiamo segnalare alcune cause, il che vuole anche dire esaminare le condizioni in cui la tutela si esercita. Prima di tutto una semplice divisione tra il numero di oggetti oggi vincolati e quello degli addetti dimostra che non è possibile che quell'importante compito sia svolto assicurando le condizioni preliminari, cioè la cura, la messa in sicurezza degli oggetti sotto vari profili; la situazione è molto varia negli specifici campi in cui quei compiti si esercitano, ma anche in quello che appare in migliori condizioni, cioè la conservazione dell'immenso patrimonio pittorico e scultoreo, accanto all'esempio dei grandi e piccoli musei dove le opere sono custodite e nei quali si svolgono importanti attività di valorizzazione e per la conoscenza diffusa fra il pubblico, attività scientifiche di rilievo, rimangono problemi molto rilevanti per il patrimonio immagazzinato, ignoto ai più, che potrebbe (non mancano questioni di natura scientifica) trovare sedi decentrate. $\mathrm{Ma}$ accanto a questo sussiste il problema di quanto sia diffuso sul territorio, spesso in condizioni non soddisfacenti, sia dal punto di vista della conservazione sia dal punto di vista del pericolo dei vandalismi ma soprattutto per la perdita del contesto, fisico e culturale. In tema di archeologia basterà far cenno al problema della manutenzione di quanto scavato, della catalogazione scientifica dei pezzi ritrovati e ricordare che la situazione è tale che si sono anche manifestate richieste, molto fondate, per la sospensione delle attività di scavo. Il patrimonio architettonico è quello che risulta meno protetto: ciascuno di noi conosce più di un edificio di grande rilevanza architettonica abbandonato; abbiamo sofferto la distruzione di interi centri antichi, ingenti e continue quelle dell'edilizia definita, a torto, minore, per la quale non si è trovata una definizione critica corretta ed esaustiva, che non soltanto è abbattuta per fenomeni di ordine speculativo, in genere per il recupero della rendita di posizione, ma anche per ignoranza della possibilità di intervento, di adeguamento alle esigenze di vita di oggi in forme rispettose e minimamente distruttive.

Possiamo constatare anche la pessima organizzazione della tutela sotto il profilo della manutenzione, della prevenzione del degrado, perché non sussiste un programma di intervento che tenga conto delle effettive urgenze sul territorio nazionale ed all'interno delle regioni. Tutti conosciamo edifici su cui si sono fatti interventi costosi dopo un lungo abbandono che ha generato condizioni di degrado irrimediabili; altrettanto diffusi i casi di spese ingiustificate, eccedenti le ordinarie 
manutenzioni in stabili in buone condizioni, mentre, anche nella ricca Lombardia, si vedono importanti testimonianze di architettura abbandonate, senza neppure il presidio della copertura o dei serramenti. Ed il capitolo della insufficienza della tutela non può non toccare il tema dei rapporti con gli attori privati del restauro: scarsi i controlli sugli interventi sulle opere d'arte mobili, poteri discrezionali nell'architettura che spesso si traducono in improprie funzioni progettuali, imponendo criteri ed orientamenti che in realtà possono essere contraddetti da altri con uguale dignità culturale, inoppugnabile il fatto che non siamo di fronte a scelte per le quali si possano dare indicazioni meccaniche. Una strada, indicata già da molti anni ma mai attuata, sarebbe quella di ridurre la discrezionalità normando non già il metodo di intervento ma la quantità e la qualità degli elaborati da sottoporre ad un controllo che consenta di verificare i temi e i problemi esaminati, le soluzioni approntate e la loro giustificazione tecnica e culturale. Impossibile normare il progetto, ma possibile normare la sua redazione: a scala edilizia, paesaggistica, territoriale.

Una prima constatazione, che sarà unita ad altre, è che la vastità del problema richiederebbe una diffusione della tutela sul territorio, ben oltre quel sovrintendente condotto che già a fine Ottocento qualcuno reclamava. E' stupefacente che lo stato abbia delegato ampi compiti in questo campo senza assicurarsi prima che vi fossero strutture adeguate al compito: le vicende in Lombardia, al momento del passaggio alla Regione delle compente di tutela ambientale, furono comiche se non tragiche ed occorsero alcuni anni prima che si formasse una struttura organizzata ed altri ancora prima che si raggiungesse un livello adeguato ai compiti (e non è il caso peggiore in Italia). Così molte competenze sono state delegate dalla Regione ai Comuni senza che si sia provveduto ad una adeguata diffusione di una cultura della tutela. Sarebbe stato possibile istituire corsi (non brevi!) per ottenere in ogni comune o quartiere delle grandi città competenze diffuse sul territorio, supporto alle autorità centrali di controllo, in grado di fornire conoscenze alle amministrazioni locali in relazione a quanto loro delegato. Già in passato la rete di ispettori onorari costituiva una importante fonte di informazioni dalla periferia al centro e viceversa, punti di riferimento preziosi, non senza qualche inconveniente che poteva essere facilmente superato.

Un altro punto che mi sembra debba essere messo alla nostra attenzione è il fatto che laddove si prendono decisioni di natura economica, di pianificazione del territorio, si producono strumenti urbanisti- 
ci esecutivi, là dovrebbe essere presente l'organizzazione della tutela che spesso viene esercitata quando la distruzione o la decontestualizzazione sono la fase finale, la conseguenza di condizioni poste all'intorno che hanno creato problemi non risolubili. Un provvedimento fiscale può produrre condizioni favorevoli alla conservazione o distruzioni più ampie di quanto qualsiasi ufficio possa ottenere o evitare, si pensi, per esempio, alle imposte sugli immobili a prescindere dal loro reddito che ha prodotto migliaia di casi di abbandono, specialmente fra gli edifici rurali, con la perdita di suppellettili, decorazioni murali, testimonianze di attività lavorative. Si noti che con questo non è necessariamente affermato che l'imposta sia iniqua o che abbia effetti esclusivamente negativi, ma è assolutamente deplorevole che questo aspetto della questione non sia emerso nella discussione di merito, che non si siano levate voci da parte degli organismi di tutela, se non per iniziativa di associazioni private che non hanno avuto ascolto. L'esempio chiarisce anche che cosa si intende quando qui si afferma che le scelte intorno alla tutela, ed ancor più all'intervento restaurativo o conservativo, siano fondamentalmente di natura politica. Il tema sembra riguardare la sola architettura, ma non è così perché investe quella della collocazione delle opere d'arte mobili, delle pitture murali, che, quando il lor destino è favorevole, sono musealizzate, cioè poste in quella situazione che, già da un secolo, era stata definita dai pessimisti la prigione dell'arte e dagli ottimisti il suo ospedale, o ricovero di anzianità.

A prescindere da qualsiasi volontà di ampliamento degli interessi di tutela che con il vincolo diretto od indiretto che riguarda milioni di oggetti, la manutenzione di quanto è proprietà degli enti pubblici è già sufficiente a generare cifre insostenibili per i bilanci, e questo vale soprattutto per gli enti locali. Inoltre non è concettualmente corretto che un bene in mano privata, e sono moltissimi quelli di grande rilevanza sotto ogni profilo, non riceva lo stesso grado di attenzione: se la conservazione risponde ad un interesse pubblico, se il bene è riconosciuto nei suoi valori culturali tanto da limitare la libera disponibilità del proprietario, non sembra equo che gli oneri siano totalmente a suo carico.

Queste considerazioni portano a una conclusione molto semplice ma ricca di conseguenze: non è possibile immaginare una tutela "passiva" dei beni culturali destinando una quota del reddito nazionale, una parte accantonata per un soddisfacimento di interessi che possano apparire se non marginali comunque estranei a ciò che si comprende comunemente nell'economia. Occorrerebbe invece che i processi eco- 
nomici si volgessero sempre più verso lo scambio di servizi di natura culturale, che conservazione e fruizione del patrimonio materiale, che è però testimone e veicolo di un significato immateriale, venissero considerati fattori di primo piano della produzione e dello scambio e contribuissero a superare la distinzione tra lavoro produttivo e realizzazione della persona. Si tratta spesso della sempre più ridotta necessità di lavoro umano, per i processi di meccanizzazione un tempo, di informatizzazione oggi; molti i sociologi indicano nella incentivazione delle attività creative una possibile soluzione, nella produzione di beni e servizi immateriali ciò che può mantenere in essere gli scambi, nel contempo consentendo ad un numero sempre maggiore di persone l'espressione della personalità: in questa direzione la rilevanza della presenza dei beni culturali può avere una importanza fondamentale. Il bene culturale come bene economico non soltanto perché provoca, per esempio, attività turistica, perché taluni di essi si comprano e vendono, perché si organizzano mostre ed eventi, ma anche come bene che soddisfa in modo immediato un fine dell'economia, la realizzazione della persona.

In questa prospettiva il rapporto collaborativo tra Stato e strutture private, comunque auspicabile, è semplicemente inevitabile, perché le persone devono essere i reali protagonisti; perché le risorse finanziarie private sono indispensabili; si deve realizzare su di un piano in cui l'ente pubblico non rinunci a garantire il rispetto dei valori di cultura ma senza monopoli di scelte di indirizzo, un tentativo reiterato, fallito persino in epoca fascista: una impostazione etica ma non in uno stato etico.

Generare un più ampio "mercato" per i beni culturali, incentivare la formazione di una forte domanda della loro fruizione, sempre più elevata sul piano qualitativo, senza che, come accade spesso, si tenda a falsi concetti di "valorizzazione" (la prima vera valorizzazione è la conoscenza del bene, il che presuppone la sua conservazione) per massimizzare i flussi di denaro ed aderire a modelli di tipo consumistico. Questo comporta anche che una politica per beni culturali, come quella per l'integrità dell'ambiente fisico, non può avere dimensioni settoriali, ma deve invece coinvolgere tutti gli aspetti della vita associata, deve provocare una crescita culturale coinvolgente e partecipata, svolgersi su diversi piani in rapporto alla consapevolezza dei fruitori, senza sacrificare gli aspetti scientifici. 